\title{
OPEN BEAUTY PRODUCTION AT HERA
}

\author{
J. KROSEBERG \\ (on behalf of the H1 and ZEUS collaborations) \\ Physik-Institut der Universität Zürich \\ Winterthurer Str. 190, CH-8057 Zürich, Switzerland \\ E-mail: Juergen.Kroseberg@desy.de
}

\begin{abstract}
Recent results on open beauty production in positron-proton collisions at a centreof-mass energy of $300 \mathrm{GeV}$ are presented. The beauty photoproduction cross section is measured by ZEUS and H1 via the semileptonic decay. Both experiments use the transverse lepton momentum with respect to a jet as an observable. The H1 central silicon vertex detector makes it possible to analyse, in addition, the lepton impact parameter spectrum, thus exploiting lifetime information for the first time at HERA. The combined lifetime and transverse momentum signature is further used to observe beauty production for the first time in deep inelastic scattering. All results are compared with NLO QCD calculations.
\end{abstract}

\section{Introduction}

The production of heavy quarks at HERA provides a valuable tool to probe proton structure and the mechanism of the hard subprocesses underlying $e p$ interactions. Within the framework of QCD, heavy quark production is predominantly gluon induced, which allows the gluon content of the proton to be investigated. The leading-order ( $\mathrm{LO}$ ) process is boson gluon fusion, where a photon emitted by the positron and a gluon coming from the proton form a quark-antiquark-pair. The exchange of a quasi-real photon (photoproduction, $\gamma p$ ) dominates over large photon virtualities (deep inelastic scattering, DIS). Perturbative QCD (pQCD) calculations, assuming the $b$ quark to be dynamically produced in the hard subprocess (massive approach), are available in next-to-leading order (NLO). In the case of beauty production, pQCD predictions are expected to be particularly reliable because the $b$ quark mass sets a hard scale. For this reason, $b$ production is considered an excellent testing ground for $\mathrm{pQCD}$ even though the cross section is two and three orders of magnitude below charm production and total cross sections, respectively. The first measurement of open $b$ photoproduction at HERA 1 yielded a cross section significantly above the QCD expectation. A similar discrepancy betweep data and theoretical prediction has been observed in $p p^{2}$ and, recently, in $\gamma \gamma{ }^{3}$ interactions. New data and improved experimental tools have now become available to further study $b$ production processes at HERA. Recent analyses, based on semileptonic $b$ hadron decays within jets, are presented here. 


\section{ZEUS}

ZEUS has measured $b$ photoproduction in inclusive muon 4 and electron analyses. Here only the electron analysis is discussed, which has recently been published: From data collected during 1996 and 1997, which correspond to an integrated luminosity of $\mathcal{L}=38.5 \mathrm{pb}^{-1}$, a $\gamma p$ sample is obtained by rejecting events in which the scattered positron is found in the main detector. Jets are reconstructed, and events with at least two jets with transverse energy $E_{t}\left(\right.$ jet $\left._{1(2)}\right)>7(6) \mathrm{GeV}$ are selected. Electrons are identified by combining calorimeter and drift chamber information. The hadronic background is statistically subtracted using the specific energy loss in the drift chamber. Backgrounds from Dalitz decays and photon conversions are removed explicitly, resulting in a sample of $(943 \pm 69)$ electrons with a transverse momentum $p_{t}>1.6 \mathrm{GeV}$ and pseudorapidity $|\eta|<1.1$. For each electron, the transverse momentum $p_{t}^{r e l}$ relative to the closest jet is reconstructed. Due to the high $b$ mass a harder $p_{t}^{r e l}$ spectrum is expected for the signal compared to the background. The measured differential cross section, $d \sigma / d p_{t}^{r e l}$, is shown in figure 1 (a), together with the prediction for beauty and charm production from the LO Monte Carlo program HERWIGG, including contributions from resolved $\gamma$ processes. Taking the normalisation from the data, a maximum likelihood fit results in a beauty fraction $f_{b}=[14.7 \pm 3.8($ stat. $)] \%$, which translates into a visible cross section of $\left[24.9 \pm 6.4(\text { stat. })_{-7.3}^{+4.2}(\right.$ syst. $\left.)\right] \mathrm{pb}$. An extrapolation to the parton level for the region $p_{T}^{b}>5 \mathrm{GeV},\left|\eta^{b}\right|<2$, photon virtuality $Q^{2}<1 \mathrm{GeV}^{2}$ and inelasticity $0.2<y<0.8$ gives

$$
\sigma_{\gamma p}^{\text {ext }}=\left[1.6 \pm 0.4(\text { stat. })_{-0.5}^{+0.3}(\text { syst. })_{-0.4}^{+0.2}(\text { extrapolation })\right] \mathrm{nb} \text {, }
$$

somewhat above the result of a NLO QCD calculation, 6 cf. figure 1 (b).
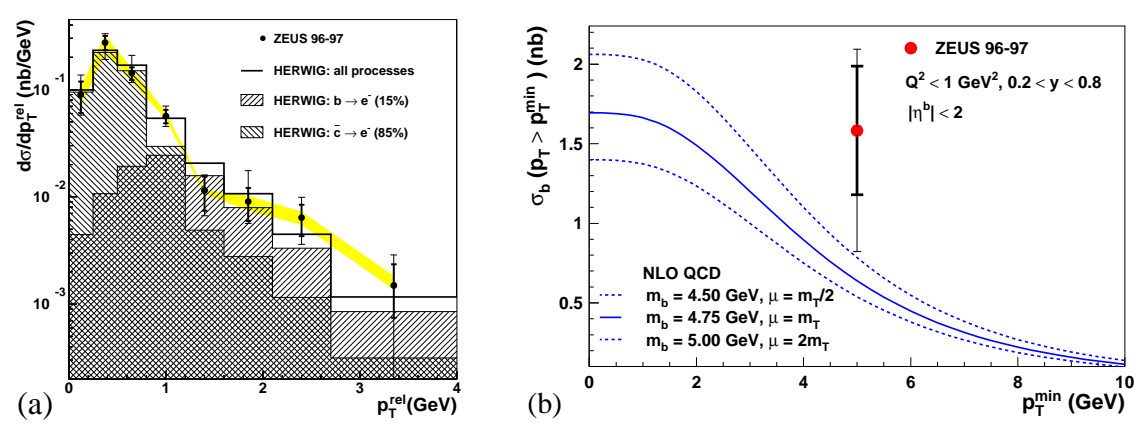

Figure 1. (a) $d \sigma / d p_{t}^{r e l}$ for data and MC (normalised and fitted to the data).

(b) Extrapolated $\sigma$ from data and NLO QCD (as a function of the minimum $p_{t}^{b}$ ). 


\section{$3 \mathrm{H} 1$}

The first H1 measurement of open $b$ production 1 was based on an inclusive muon $p_{t}^{r e l}$ analysis using $1996 \gamma p$ data. In the new analysis, the $\mathrm{H} 1$ central silicon tracker CST 8 is used to detect $b$ hadrons also via their long lifetimes. In a sample of dijet $\gamma p$ events $\left(E_{t}\left(\right.\right.$ jet $\left.\left._{1,2}\right)>5 \mathrm{GeV}\right)$ collected in 1997 $\left(\mathcal{L}=14.7 \mathrm{pb}^{-1}\right), 1415$ muon candidates are selected, which are identified in the instrumented iron and the central drift chamber and precisely measured in the CST. The decomposition of the sample is obtained from a likelihood fit to the muon signed impact parameter $(\delta)$ spectrum, 1 which at large positive values is expected to be signal dominated. Contributions from beauty and charm production are modelled by the AROMA MC program 9 based on LO QCD and parton showers. Light quark background, dominated by misidentified hadrons (fake muons), is selected from data. The fit describes the data well, cf. figure 2(a), and yields a signal contribution of $f_{b}=(26 \pm 5$ (stat.)) $\%$. Translating this into a visible cross section, the previous $\mathrm{H} 1$ measurement is confirmed using new data and an independent signature.

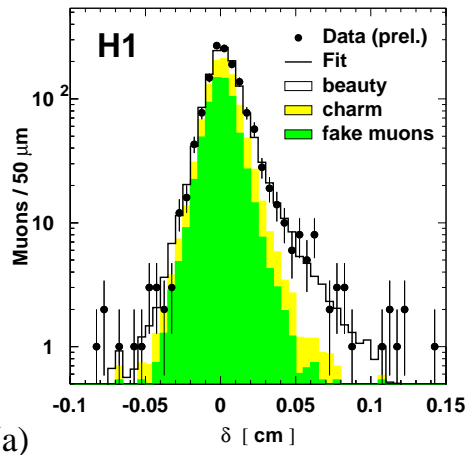

(a)

Figure 2. (a) Muon $\delta$ distribution ( $\gamma p$ sample) with the decomposition from the $\delta$ fit. (b) $p_{T}^{r e l}$ spectrum for the DIS sample, with the result of the combined $\left(\delta, p_{T}^{r e l}\right)$ fit.

After having verified the consistency of $p_{t}^{r e l}$ and $\delta$, the separation power of both observables is combined in a fit to the two-dimensional $\left(\delta, p_{T}^{r e l}\right)$ distribution. The resulting cross section in the visible range, defined by $p_{t}(\mu)>2 \mathrm{GeV}, 35^{\circ}<\theta(\mu)<130^{\circ}, Q^{2}<1 \mathrm{GeV}^{2}$ and $0.1<y<0.8$, is

$$
\sigma_{\gamma p}^{v i s}=[160 \pm 16 \text { (stat.) } \pm 29 \text { (syst.) }] \mathrm{pb} .
$$

${ }^{a} \delta$ is reconstructed in the plane perpendicular to the beam. $|\delta|$ is the distance of closest approach of the candidate track to the event primary vertex. The sign is positive if the track crosses the jet axis downstream with respect to the vertex, and negative otherwise.

DIS01-proceedings: submitted to World Scientific 
The combination of published and new results yields a visible cross section of $[170 \pm 25] \mathrm{pb}$. A NLO QCD calculation with the FMNR program gives a significantly lower result of [54 \pm 9$]$ pb. 1

Having established the new method in the photoproduction regime, it is further used to measure for the first time the open $b$ production cross section in DIS. By reconstructing the scattered beam positron in the backward calorimeter, a DIS event sample is obtained, which corresponds to an integrated luminosity of $\mathcal{L}=10.5 \mathrm{pb}^{-1}$. After applying the same muon and jet selection as in the photoproduction analysis and restricting the kinematic range to $2 \mathrm{GeV}^{2}<Q^{2}<100 \mathrm{GeV}^{2}$ and $0.05<y<0.7,171$ muon candidates remain. The combined $\left(\delta, p_{T}^{\text {rel }}\right)$ fit results in $f_{b}=(43 \pm 8($ stat. $)) \%$ and gives a good description of the data; for the $p_{T}^{r e l}$ projection this is shown in figure $2(\mathrm{~b})$. The corresponding visible cross section is

$$
\sigma_{\mathrm{DIS}}^{\text {vis }}=[39 \pm 8 \text { (stat.) } \pm 10 \text { (syst.) }] \mathrm{pb} .
$$

This again exceeds significantly the $\mathrm{NLO}$ QCD prediction of $(11 \pm 2) \mathrm{pb}$ obtained from the HVQDIS program.10

\section{Summary}

Open beauty production is measured in inclusive lepton analyses by ZEUS and H1 using new data and including the $b$ lifetime signature. Previous photoproduction results are confirmed and improved, and the DIS cross section is measured for the first time. All measured cross sections are found to be above the NLO QCD predictions. For the H1 results the excess is significant.

\section{References}

1. H1 Collab., C. Adloff et al., Phys. Lett. B 467, 156 (1999) and erratum (to be published).

2. CDF Collab., F. Abe et al., Phys. Rev. D 53, 1051 (1996).

D0 Collab., S. Abachi et al., Phys. Lett. B 370, 239 (1996).

3. L3 Collab., M.Acciarri et al., Phys. Lett. B 503, 10 (2001).

OPAL Collab., OPAL Physics Note PN455 (2000).

4. ZEUS Collab., ICHEP 1999, Tampere, Finland, contrib. paper no. 498.

5. ZEUS Collab., J. Breitweg et al., Eur. Phys. J. C 18, 625 (2001).

6. G. Marchesini et al. Comp. Phys. Commun. 67, 465 (1992).

7. S. Frixione et al., Phys. Lett. B 348, 633 (1995).

8. D. Pitzl et al., Nucl. Instrum. Methods A 454, 334 (2000).

9. G. Ingelman et al., Comp. Phys. Commun. 101, 135 (1997).

10. B.W. Harris and J. Smith, Phys. Rev. D 57, 2806 (1998). 